\title{
Preoperative serum lactate measurement as a prognostic biomarker for patients with primary brain tumor
}

Chung-Chih Shih, Tzong-Shiun Lee, Pei-Lin Lin, Hsiao-Liang Cheng, Chun-Yu Wu Department of Anesthesiology, National Taiwan University Hospital, Taipei, Taiwan

\section{Background}

High grade brain tumor is one of the most malignant cancer. The accurate diagnosis of high grade brain is sometimes difficult. Currently, there are several candidate for brain tumor or brain injury including glial neuron-specific biomarkers fibrillary acidic protein (GFAP), S100B, neuron-specific enolase (NSE), and neutrophil gelatinase-associated lipocalin (NGAL). In addition,

Ramamani et al. demonstrated that serum lactate levels can potentially be used as a non-invasive biomarker of glioma which correlates with brain tumor grade. The goals of this study were

(1) to determine which serum biomarker could serve as the diagnostic or prognostic biomarker for patients with primary brain tumor undergoing brain tumor resection, and (2) to determine the independent factor associated with high grade brain tumor.

\section{Method}

This study was conducted by retrospectively analysis of the data from a previous study investigating intraoperative goaldirected therapy for supratentorial brain tumor resection in

NTUH, between 2013 and 2015.

Data of 74 patients including patient demographics, comorbidities, tumor pathology, World Health Organization (WHO) grading of the tumor were analyzed (Table 1).

pretreatment serum biomarkers, including lactate, lactate dehydrogenase, glial fibrillary acidic protein, S100ß, neuronspecific enolase, and neutrophil gelatinase-associated lipocalin

were measured and compared with postoperative outcomes including tumor grading, progression-free survival and overall survival.

Table 1. Patient characteristics in study cohort

\begin{tabular}{|c|c|c|c|c|}
\hline Characteristic & $\begin{array}{l}\text { Total } \\
(\mathrm{N}=74)\end{array}$ & $\begin{array}{l}\text { High grade } \\
\qquad(\mathrm{N}=20)\end{array}$ & $\begin{array}{r}\text { Low grade } \\
(\mathrm{N}=54)\end{array}$ & $P$ Value \\
\hline Age (years) & $56.55 \pm 11.4$ & $54.2 \pm 12.5$ & $57.4 \pm 10.9$ & 0.293 \\
\hline Male no. (\%) & $23(31.1)$ & $8(40.0)$ & $12(27.8)$ & 0.398 \\
\hline Body weight (kg) & $60.40 \pm 9.6$ & $58.9 \pm 8.7$ & $60.9 \pm 9.9$ & 0.455 \\
\hline \multicolumn{5}{|l|}{ Comorbidities, No. (\%) } \\
\hline Cadiovascular disease & $6(8.1)$ & $2(10.0)$ & $4(7.4)$ & 0.659 \\
\hline Hypertension & 14 (18.9) & $5(25.0)$ & $9(16.7)$ & 0.308 \\
\hline Diabetes & $7(9.5)$ & $3(15.0)$ & $4(7.4)$ & 0.379 \\
\hline Other cancer & $5(6.8)$ & $1(5.0)$ & $4(7.4)$ & 0.589 \\
\hline Tumor size (cm) & $4.39 \pm 1.75$ & $4.50 \pm 1.78$ & $4.31 \pm 1.73$ & 0.450 \\
\hline \multicolumn{5}{|l|}{ Serium biomarkers } \\
\hline Lactate (mmol/L) & $1.38 \pm 0.92$ & $1.91 \pm 1.06$ & $1.19 \pm 0.78$ & 0.011 \\
\hline $\mathrm{LDH}(\mathrm{mU} / \mathrm{ml})$ & $38.22 \pm 36.73$ & $39.6 \pm 31.28$ & $37.7 \pm 38.8$ & 0.401 \\
\hline NGAL (ng/ml) & $0.483 \pm 0.544$ & $0.544 \pm 0.535$ & $0.465 \pm 0.554$ & 0.516 \\
\hline GFAP (mg/L) & $0.243 \pm 0.483$ & $0.14 \pm 0.35$ & $0.28 \pm 0.52$ & 0.097 \\
\hline NSE (ng/ml) & $15.22 \pm 54.79$ & $10.77 \pm 14.67$ & $16.86 \pm 63.62$ & 0.339 \\
\hline $\mathrm{S} 100 \beta(\mathrm{pg} / \mathrm{ml})$ & $87.06 \pm 128.95$ & $93.61 \pm 131.76$ & $84.59 \pm 129.06$ & 0.353 \\
\hline
\end{tabular}

Figure 1. Receiver operating characteristics analysis of serum lactate in discriminating high grade from low grade brain tumors

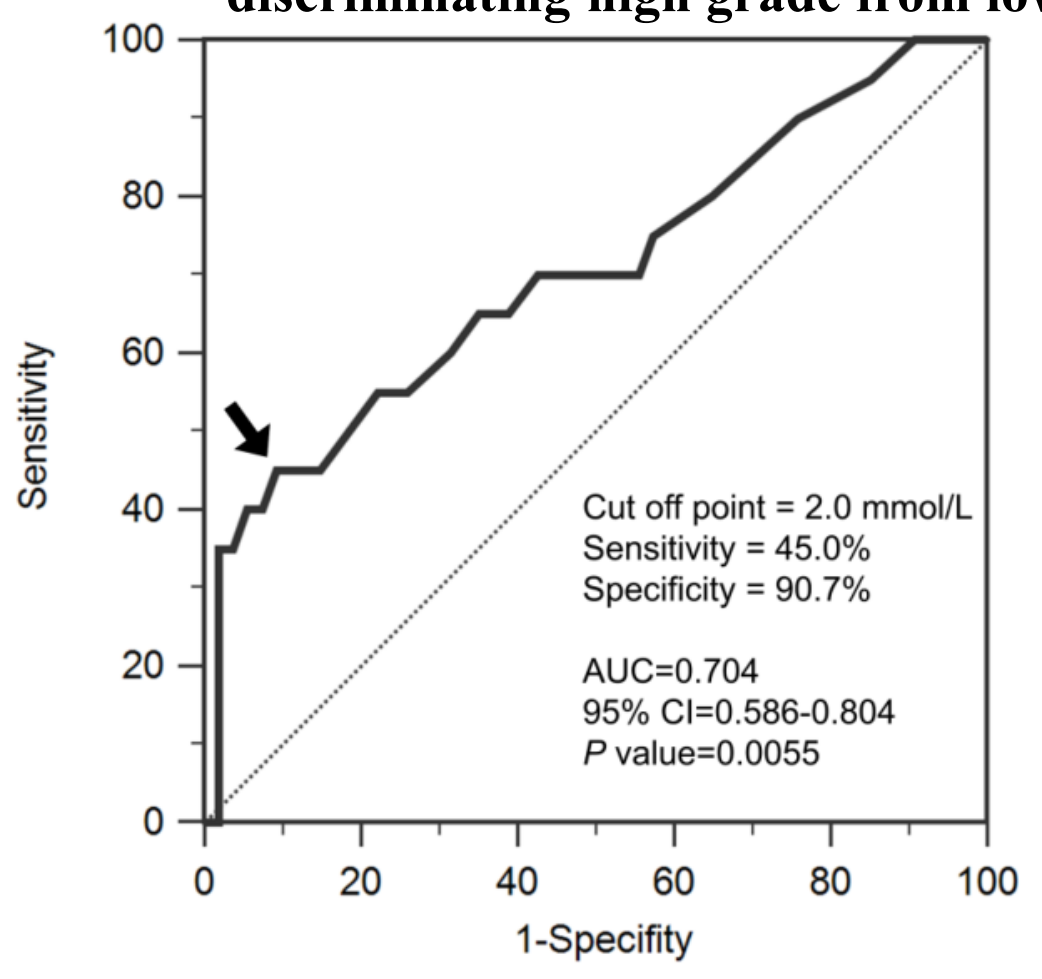

\section{Result}

1.Multivariate Logistic regression revealed that only elevated serum lactate is the risk factor for high grade brain tumor

$$
(p=0.0018) \text {. }
$$

2.Kaplan-Meier survival curves show significant difference in progression-free survival (PFS) time between patients with high or low lactate $(\mathrm{p}=0.021$, table 3$)$, but no significant difference in

$$
\text { survival (OS) }(p=0.093)
$$

3.A multivariateoverall analysis showed higher lactate is associated with poor OS $(p=0.048)$. Higher lactate and male are

factors associated with poor PFS $(p=0.021, p=0.023$, respectively, table 2 )

Figure 2. Kaplan-Meier survival analysis of serum lactate measurement in association with patient survival.
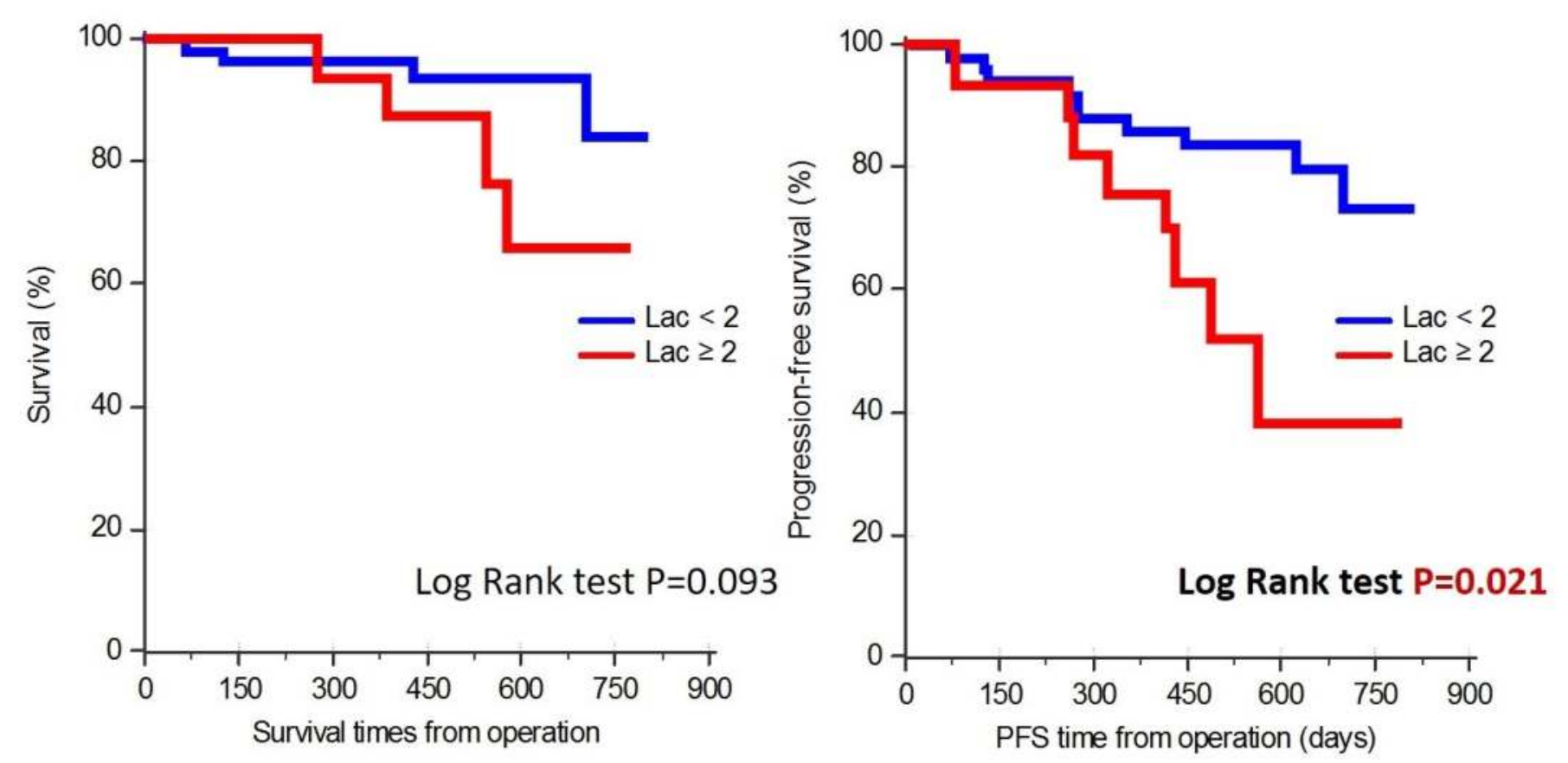

Table 3. Univariate and multivariate cox regression analyses of overall

\begin{tabular}{|c|c|c|c|c|c|c|}
\hline \multirow{3}{*}{ Factor } & & & & & & \\
\hline & \multicolumn{3}{|c|}{ univariate analysis } & \multicolumn{3}{|c|}{ Multivariate analysis } \\
\hline & HR & $95 \% \mathrm{Cl}$ & $P$ Value & HR & $95 \% \mathrm{Cl}$ & $P$ Value \\
\hline Age (years) & 1.041 & $0.973-1.114$ & 0.247 & - & & \\
\hline Gender (male:female) & 4.349 & $1.037-18.247$ & 0.045 & 3.785 & $0.903-15.859$ & 0.0686 \\
\hline Body weight (kg) & 0.994 & $0.925-1.070$ & 0.881 & - & & \\
\hline Tumor size $(\mathrm{cm})$ & 1.214 & $0.835-1.763$ & 0.310 & - & & \\
\hline \multicolumn{7}{|l|}{ Serium biomarkers } \\
\hline Lactate (mmol/L) & 2.046 & $1.078-3.886$ & 0.029 & 2.049 & $1.0057-4.1747$ & 0.0482 \\
\hline $\mathrm{LDH}(\mathrm{mU} / \mathrm{mL})$ & 1.013 & $0.998-1.028$ & 0.084 & - & & \\
\hline $\operatorname{NGAL}(\mathrm{ng} / \mathrm{mL})$ & 2.987 & $1.067-8.363$ & 0.037 & - & & \\
\hline GFAP (mg/L) & 0.983 & $0.169-5.704$ & 0.985 & - & & \\
\hline $\mathrm{NSE}(\mathrm{ng} / \mathrm{mL})$ & 0.936 & $0.867-1.069$ & 0.478 & - & & \\
\hline $\mathrm{S} 100 \beta(\mathrm{pg} / \mathrm{mL})$ & 0.997 & $0.987-1.006$ & 0.490 & - & & \\
\hline
\end{tabular}
survival and progression-free survival in primary brain tumor patients

\begin{tabular}{|c|c|c|c|c|c|c|}
\hline \multirow{3}{*}{ Factor } & \multicolumn{6}{|c|}{ PFS } \\
\hline & \multicolumn{3}{|c|}{ univariate analysis } & \multicolumn{3}{|c|}{ Multivariate analysis } \\
\hline & HR & $95 \% \mathrm{Cl}$ & $P$ Value & HR & $95 \% \mathrm{Cl}$ & $P$ Value \\
\hline Age (years) & 1.0290 & $0.985-1.076$ & 0.195 & - & & \\
\hline Gender (male:female) & 3.0190 & $1.192-7.645$ & 0.020 & 2.951 & $1.161-7.496$ & 0.0229 \\
\hline Body weight (kg) & 1.0110 & $0.965-1.059$ & 0.647 & - & & \\
\hline Tumor size (cm) & 1.1230 & $0.868-1.453$ & 0.378 & - & & \\
\hline \multicolumn{7}{|l|}{ Serium biomarkers } \\
\hline Lactate (mmol/L) & 1.6190 & $1.079-2.428$ & 0.020 & 1.658 & $1.078-2.548$ & 0.0211 \\
\hline $\mathrm{LDH}(\mathrm{mU} / \mathrm{mL})$ & 1.0090 & $0.999-1.019$ & 0.067 & - & & \\
\hline NGAL (ng/mL) & 2.0620 & $0.993-4.279$ & 0.052 & - & & \\
\hline GFAP (mg/L) & 1.2600 & $0.460-3.447$ & 0.653 & - & & \\
\hline NSE (ng/mL) & 0.9980 & $0.989-1.008$ & 0.740 & - & & \\
\hline $\mathrm{S} 100 \beta(\mathrm{pg} / \mathrm{mL})$ & 1.0010 & $0.997-1.004$ & 0.733 & - & & \\
\hline
\end{tabular}

\section{Conclusion}

An elevated pretreatment serum lactate level is a prognostic biomarker of high-grade primary brain tumors and is significantly associated with poor PFS in patients with supratentorial brain tumors undergoing tumor resection. By contrast, other serum biomarkers are not significantly associated with oncological outcomes. 